International Journal of Modern Physics B Vol. 20, Nos. $30 \& 31$ (2006) 4992-5007

(C) World Scientific Publishing Company

\title{
TOWARDS A COUPLED-CLUSTER TREATMENT OF SU $(N)$ LATTICE GAUGE FIELD THEORY
}

\author{
RAYMOND F. BISHOP*, NORBERT LIGTERINK ${ }^{\dagger}$ and NIELS R. WALET \\ School of Physics and Astronomy, The University of Manchester, \\ Sackvile Street Building, P.O. Box 88, Manchester M60 1QD, UK \\ *raymond.bishop@manchester.ac.uk
}

\begin{abstract}
A consistent approach to Hamiltonian $\mathrm{SU}(N)$ lattice gauge field theory is developed using the maximal-tree gauge and an appropriately chosen set of angular variables. The various constraints are carefully discussed, as is a practical means for their implementation. A complete set of variables for the colourless sector is thereby determined. We show that the one-plaquette problem in $\mathrm{SU}(N)$ gauge theory can be mapped onto a problem of $N$ fermions on a torus, which is solved numerically for the low-lying energy spectra for $N \leq 5$. We end with a brief discussion of how to extend the approach to include the spatial (inter-plaquette) correlations of the full theory, by using a coupled-cluster method parametrisation of the full wave functional.
\end{abstract}

Keywords: Gauge fixing; maximal-tree gauge; Hamiltonian approach.

\section{Introduction}

Largely due to its conceptual elegance and simplicity, most calculations in lattice gauge-field theory (LGFT) have been performed within the Lagrangian formulation, which is based on a path-integral approach to the imaginary-time propagator. A great deal of work has been done to improve the accuracy of the Monte Carlo methods used in the numerical implementation of the Lagrangian approach, further increasing its viability.

Nevertheless, the much less studied Hamiltonian approaches have at least four important advantages. Firstly, since it is based on an imaginary-time evolution, the Lagrangian approach does not allow easy access to the vacuum wave functional. By contrast, such a wave functional is at the core of any Hamiltonian approach. Once the wave functional is known, most important properties of theories such as QCD, including, for example, confinement and chiral symmetry, should follow automatically. Secondly, time-dependent phenomena can only be discussed in a real-time (Hamiltonian) setting. Thirdly, the physical interpretation of the variables is much more transparent in the Hamiltonian framework, where, for example, electric and magnetic operators have their classical meanings. Lastly, the Hamiltonian approach

${ }_{\dagger}$ Present address: Universiteit Twente, Faculteit EWI/Elektrotechniek, Postbus 217, 7500 AE Enschede, The Netherlands. 
may be studied both with analytical techniques and numerically. Thus, for example, we can study low-lying excitations with a harmonic approximation and we can disentangle the dependence of observables on the parameters in the approximation.

In view of these advantages, we develop here a consistent approach to Hamiltonian LGFT using the maximal-tree gauge, which is further formulated in terms of a set of angular variables. The various constraints in the theory are discussed and implemented, and an independent and complete set of variables is determined for the colourless sector. We describe a general scheme to construct the eigenstates of the electric energy operator using an efficient symbolic method. It is shown how the one-plaquette problem for $\mathrm{SU}(N)$ LGFT can be mapped onto an $N$-fermion problem for arbitrary values of $N$. The low-lying energy spectra are investigated numerically, and explicit solutions are shown for $\mathrm{SU}(2), \mathrm{SU}(3), \mathrm{SU}(4)$, and $\mathrm{SU}(5)$. Previous attempts to use the coupled cluster method to include multi-plaquette correlations have largely been confined to the ground-state energy and low-lying excitation gaps (glueball masses) in the pure gauge (gluon) sector (i.e., without fermions) of the $\mathrm{U}(1)$ and $\mathrm{SU}(2)$ cases in one and two spatial dimensions. ${ }^{1,2} \mathrm{We}$ conclude with a brief discussion on how the new maximal-tree formulation might profitably be used to make further progress for the non-Abelian $\mathrm{SU}(N)$ gauge theories with $N \geq 2$ in three spatial dimensions where, as we show, significant new complications arise.

\section{Hamiltonian Lattice Gauge Theory}

The original Abelian gauge theory of electromagnetism was extended by Yang and Mills $^{3}$ using gauge fields of more complicated structure, which included internal degrees of freedom. This generates self-interactions since the gauge fields do not commute, but are chosen to obey the commutation relations of a specific Lie algebra.

\subsection{Lagrangian}

We shall concentrate on a gauge-field Lagrangian where the field $A_{\mu}$ is an element of the Lie algebra $\operatorname{su}(N)$,

$$
\mathcal{L}=\frac{1}{2 g^{2}} \operatorname{Tr}\left[F_{\mu \nu} F^{\mu \nu}\right]
$$

Here the field tensor $\boldsymbol{F}_{\mu \nu}$ is defined as

$$
\boldsymbol{F}_{\mu \nu} \equiv \partial_{\mu} \boldsymbol{A}_{\nu}-\partial_{\nu} \boldsymbol{A}_{\mu}-i\left[\boldsymbol{A}_{\mu}, \boldsymbol{A}_{\nu}\right],
$$

and the field variable $A_{\mu}$ is an element of the algebra, conveniently parametrised as

$$
A_{\mu} \equiv g \frac{1}{2} \lambda^{a} A_{\mu}^{a}
$$

The $\lambda^{a}$ are the $N^{2}-1$ generators of the Lie algebra, satisfying the commutation relations

$$
\left[\lambda^{a}, \lambda^{b}\right]=2 i f^{a b c} \lambda^{c}
$$


The index $a$ thus runs from 1 to $N^{2}-1$. The $\lambda^{a}$ can be represented by traceless $N \times N$ matrices, normalised such that their squares have trace 2 , as can be seen from the anticommutation relations

$$
\left\{\lambda^{a}, \lambda^{b}\right\}=2 d^{a b c} \lambda^{c}+\frac{4}{N} \delta_{a b} I
$$

In Eq. (3) we have absorbed the coupling constant $g$ into the field $A_{\mu}$, so that we can interpret the fields geometrically, since the field tensor is now the curvature that follows from the covariant derivative

$$
\boldsymbol{d}_{\mu} \equiv \partial_{\mu}-i\left[\boldsymbol{A}_{\mu}, \cdot\right]
$$

from which follows the relation

$$
\left[\boldsymbol{d}_{\mu}, \boldsymbol{d}_{\nu}\right]=-i\left[\boldsymbol{F}_{\mu \nu}, \cdot\right]
$$

As we are interested in the Hamiltonian, we perform the standard equal-time quantisation and reformulate the Lagrangian in terms of generalised electric and magnetic fields. This is strictly speaking a 3 -dimensional result, since this interpretation requires the use of three-dimensional algebra. We shall nonetheless use the result below for other numbers of spatial dimensions as well. We find

$$
\mathcal{L}=\frac{1}{g^{2}} \operatorname{Tr}\left[\sum_{k=1}^{D}\left(\boldsymbol{E}_{k}^{2}-\boldsymbol{B}_{k}^{2}\right)\right],
$$

where $B_{i} \equiv-\frac{1}{2} \epsilon_{i j k} F_{j k}$ and $E_{i} \equiv F_{i 0}$. Since we wish to impose the temporal gauge $\boldsymbol{A}_{0}=0$, we separate the Lagrangian into two parts, thereby isolating the $A_{0}$ dependent part, ${ }^{4}$

$$
\mathcal{L}=\frac{1}{g^{2}} \operatorname{Tr}\left[\sum_{k=1}^{D}\left(E_{k}^{2}-B_{k}^{2}\right)\right]_{A_{0}=0}+\frac{1}{g^{2}} \operatorname{Tr}\left[\boldsymbol{A}_{0} \mathcal{G}+\boldsymbol{A}_{0} \mathcal{X}\left(\boldsymbol{A}_{0}\right)\right],
$$

where we have added a total divergence. The function $\mathcal{X}$ is second order in $A_{0}$ and does not contribute to the equations of motion or to the constraint equations in the temporal gauge $\left(A_{0}=0\right)$ discussed below.

Since the time-derivative of $\boldsymbol{A}_{0}$ does not occur in the Lagrangian, equation of motion for $A_{0}$ is a time-independent algebraic equation, and thus $A_{0}$ is constant. This set of equations (one for each colour index) is the non-Abelian analogue of the Gauss' law constraint, and in the absence of colour charges they take the simple form

$$
\mathcal{G}^{a}(\mathbf{x})=0
$$

where

$$
\mathcal{G}^{a}(\mathbf{x})=\sum_{i=1}^{D}\left[\partial_{i} E_{i}^{a}(\mathbf{x})+g f^{a b c} A_{i}^{b}(\mathbf{x}) E_{i}^{c}(\mathbf{x})\right]=\sum_{i=1}^{D} \boldsymbol{d}_{i} E_{i}^{a}
$$


The components of the fields can be obtained via the relation

$$
A_{\mu}^{a}=\frac{1}{g} \operatorname{Tr}\left[A_{\mu} \lambda^{a}\right] \quad, \quad E_{\mu}^{a}=\frac{1}{g} \operatorname{Tr}\left[E_{\mu} \lambda^{a}\right] .
$$

The constraints obey the same commutation relations as the generators of the gauge group. Thus, Gauss' law cannot be implemented as a strict operator condition as it leads to contradictions, since the non-commuting constraints cannot all be diagonalised simultaneously. However, within the physical (in this case colourless) subspace defined by

$$
\left.\mathcal{G}^{a}(\mathbf{x}) \mid \text { Phys }\right\rangle=0
$$

no such problem arises, since the eigenvalue of the commutators is also 0 . The space of states consists of wave functionals, taking values on the $\mathrm{SU}(N)$ group manifold. From Eq. (13) we find $N^{2}-1$ functional conditions on each wave functional, consisting of functions on the group manifold at each space point.

As is well known, quantisation of problems involving redundant degrees of freedom (i.e., where some of the equations of motion are constraints) is quite involved. The two main techniques used are Dirac and BRS quantisation, and they require a large amount of additional analysis. For more details one can consult the seminal work by Dirac, ${ }^{5,6}$ as well as Refs. $[4,7,8,9,10]$. If we are able to work within the physical subspace only, we can ignore these formal problems and define the quantisation of the canonical momenta, $\Pi_{i}^{a} \equiv \partial_{0} A_{i}^{a}$, by

$$
\Pi_{i}^{a}(\mathbf{x})=\hat{E}_{i}^{a}=-i \frac{\delta}{\delta A_{i}^{a}(\mathbf{x})},
$$

which involves a functional derivative ${ }^{11,12}$ with respect to the field variables.

Since $A_{0}^{a}$ is not dynamical, we cannot associate a canonical momentum with it. We therefore use the temporal gauge, $A_{0}^{a}=0$, which leaves us with a residual gauge freedom $\phi(\mathbf{x})$ independent of the time coordinate, such that under the transformation $A_{\mu}(\mathbf{x}) \rightarrow{ }^{\phi} A_{\mu}(\mathbf{x})$, where

$$
\begin{aligned}
\phi_{A_{\mu}}(\mathbf{x}) & =\boldsymbol{\phi}(\mathbf{x}) A_{\mu}(\mathbf{x}) \phi^{-1}(\mathbf{x})+i\left[\partial_{\mu} \phi(\mathbf{x})\right] \phi^{-1}(\mathbf{x}), \\
{ }^{\phi} F_{\mu \nu}(\mathbf{x}) & =\phi(\mathbf{x}) \boldsymbol{F}_{\mu \nu}(\mathbf{x}) \phi^{-1}(\mathbf{x}),
\end{aligned}
$$

with $\phi \in \mathrm{SU}(N)$, the Lagrangian is invariant.

\subsection{Discretization and the Hamiltonian}

Many quantum field theories suffer from singularities, both in the infrared and ultraviolet limits. In many interesting cases, such as $\mathrm{QCD},{ }^{13}$ these are renormalisable. Rather than dealing directly with the continuum, we shall regularise the problem by introducing a simple hypercubic lattice in the $D$-dimensional space, with lattice spacing $a$. Since we are pursuing a Hamiltonian approach, time will remain continuous. We shall concentrate here only on the pure gauge theory. 
As is by now well known (and see our full paper ${ }^{14}$ for further details), the system is described by a set of gauge fields (or chromo-vector-potentials)

$$
A_{i}=\frac{1}{2} g \sum_{a=1}^{N^{2}-1} \lambda^{a} A_{i}^{a},
$$

that are now defined on the links $l$ of the lattice. They are Hermitian, since $\lambda^{a}$ is Hermitian. The chromo-electric fields, $E_{l}^{a}$, are the corresponding canonical momenta obeying the commutation relation,

$$
\left[E_{l}^{a}, A_{l^{\prime}}^{a^{\prime}}\right]=-i \delta_{l l^{\prime}} \delta_{a a^{\prime}}
$$

The group elements are the link variables, $U_{l} \equiv \exp \left\{i a A_{l}\right\}$, which are thus $N \times N$ matrices. They correspond to parallel transporters on the lattice. Since $A_{l}$ is an element of the $\operatorname{su}(N)$ Lie algebra, $U_{l}$ is an $\operatorname{SU}(N)$ matrix. The product of four such group elements around a primitive square on the lattice (usually called a plaquette or Wilson plaquette) defines the corresponding plaquette operator, $U_{p} \equiv$ $U_{1} U_{2} U_{3}^{-1} U_{4}^{-1}$. Their traces form the simplest gauge-invariant quantities on the lattice.

Ignoring temporarily problems with overcompleteness of the variables, one can derive the Kogut-Susskind Hamiltonian, ${ }^{9}$

$$
\begin{aligned}
H_{\mathrm{KS}} & =\frac{g^{2}}{2 a^{D-2}}\left(\sum_{l} \sum_{a=1}^{N^{2}-1} E_{l}^{a} E_{l}^{a}+\lambda \sum_{p} \operatorname{Tr}\left[2-U_{p}-U_{\cdot p}^{-1}\right]\right) \\
& \equiv \frac{g^{2}}{2 a^{D-2}}\left(H_{E}+\lambda H_{M}\right),
\end{aligned}
$$

where $D$ is the number of spatial dimensions, and $\lambda \equiv a^{2 D-6} / g^{4}$. The sum on $l$ in the electric term $H_{E}$ runs over all links, while the sum on $p$ in the magnetic term $H_{M}$ runs over all plaquettes on the lattice.

Our physical states are now gauge-invariant in the vacuum sector. So far, the price we have paid for adopting the Hamiltonian approach is twofold. Firstly, explicit Lorentz invariance has been broken and, secondly, the problem remains of determining the physical subspace.

\subsection{A first look at handling the constraints}

As we have seen above, the gauge freedom leads to constrained dynamics. The residual gauge symmetry involves all time-independent local gauge transformations. These gauge freedoms thus generally prove an obstacle to establishing a proper set of variables in which the wave functional may be expressed. Before proceeding let us do some simple counting of the number of independent (i.e., unconstrained) degrees of freedom in our formulation. The primitive variables are the set $E_{l}^{a}$ defined on the set of $N_{l}$ links. Hence, the total number of degrees of freedom is $\left(N^{2}-1\right) N_{l}$, where the first factor is simply the number of $\mathrm{SU}(N)$ group generators. However, 
Table 1. The number of degrees of freedom on a hypercubic lattice of size $n \times n \times \cdots n$, for different numbers of space dimensions, $D$. [The $D=" 1$ " case comprises $n$ plaquettes on a line.]

\begin{tabular}{l|cccc}
\hline Dimensionality, $D$ & "1" & 2 & 3 & $D \geq 2$ \\
\hline number of sites, $N_{s}$ & $2(n+1)$ & $(n+1)^{2}$ & $(n+1)^{3}$ & $(n+1)^{D}$ \\
number of links, $N_{l}$ & $3 n+1$ & $2 n(n+1)$ & $3 n(n+1)^{2}$ & $D n(n+1)^{D-1}$ \\
number of plaquettes, $N_{p}$ & $n$ & $n^{2}$ & $3 n^{2}(n+1)$ & $\frac{1}{2} D(D-1) n^{2}(n+1)^{D-2}$ \\
\hline
\end{tabular}

there are constraints between them due to Gauss' law. Thus, we can readily derive the lattice versions $\mathcal{G}_{i}^{a}$ of the continuum generators $\mathcal{G}^{a}(\mathbf{x})$, for each of the $N_{s}$ lattice sites $i$. Hence, the number of (unconstrained) independent degrees of freedom is

$$
N_{2 b}=\left(N^{2}-1\right)\left(N_{l}-N_{s}+1\right),
$$

where the additional unity term in the second factor in this expression arises due to the overall global gauge degree of freedom that would finally still remain.

As shown in Table 1, we see that the total number of plaquettes, $N_{p}$ on the lattice is given by $N_{p}=N_{l}-N_{s}+1$ for the cases $D=1$ and $D=2$, but this relation is not true for $D>2$. The construction of Gauss' law makes it clear why it is so attractive to work with plaquette variables or, more generally, with traces of products of group operators $U_{l}$ around closed loops (i.e., Wilson loops), since these variables are automatically gauge-invariant. Hence, for $D=1,2$ the plaquette variables form a complete (i.e., neither over- nor under-complete) set of unconstrained variables. We turn below to the vexed question of what variables to choose for $D>2$.

\section{Gauge Fixing and Maximal Trees}

As we have seen above, it is the gauge freedom that leads to the constrained dynamics and the fact that the set of link variables must, in general, be over-complete. However, since the link variables are still one of the most attractive sets to use, we are motivated to fix the gauge as much as possible. We actually choose to fix the gauge fully (apart from an overall global gauge transformation that we cannot fix) by separating all of the links on the spatial lattice into two sets. One set is chosen to be just sufficient to connect any two lattice sites in a unique way. Any such set of links is called a maximal tree. ${ }^{15,16}$ Its choice for a given lattice is clearly not unique, but one such choice is shown in Fig. 1 for $D=3$. The case shown corresponds to the union of all links on the $x$-axis for $y=z=0$, and all links in the $y$-direction for $z=0$, and all links in the $z$-direction, with an obvious labelling for the axes.

Thus, all $\mathrm{SU}(N)$ link variables $U_{l}$ are thereby divided into two non-overlapping sets, $\left\{U_{l}\right\} \rightarrow\left\{V_{l} \mid l \notin\right.$ tree; $W_{l} \mid l \in$ tree $\}$. The variables $\left\{W_{l}\right\}$ are now associated with the irrelevant gauge degrees of freedom; they are essentially the irrelevant longitudinal electric fields. Conversely, the variables $\left\{V_{l}\right\}$ form our basis for the relevant degrees of freedom, the magnetic variables. However, these latter link variables are not by themselves invariant under local gauge transformations. Hence the wave 

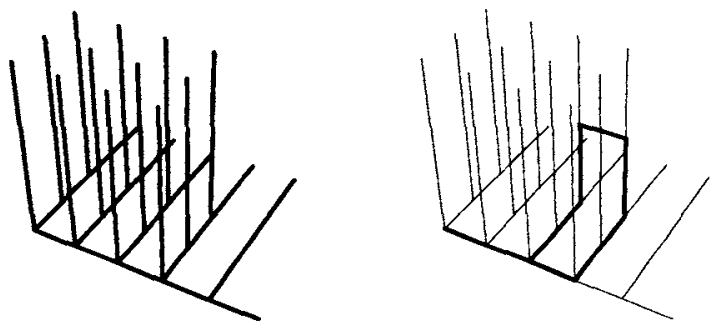

Fig. 1. A particular choice of maximal tree for $D=3$ is shown on the left; and the path associated with a typical variable $X_{l}$ for a particular link not on this tree is shown on the right.

function cannot depend directly on them since, gauge invariance would then be violated. Instead, we transform the links not on the tree into closed loops by combining them with (unique) paths on the maximal tree joining each end of the link from and to the origin, as is illustrated in Fig. 1. In this way we make a unique mapping, $V_{l} \leftrightarrow X_{l} ; \forall l \notin$ tree, where $X_{l}$ simply comprises a product of the corresponding $U_{l}$ or $U_{l}^{-1}$ operators on the links comprising the closed loop, where the inverse operators appear on links pointing back to the origin, in a convention that orients the links on the maximal tree along the direction away from the origin. Thus, all the variables $X_{l}$ transform in the same way under local gauge transformations with the gauge transformation at the origin, and are invariant under all other local gauge changes. We know that when we fix the gauge we cannot fix a global gauge transformation, and we are thus led to identify this with the one at the origin.

We have shown ${ }^{14}$ that the electric field operator $E_{l}^{a}$ associated with the link $l$ on the maximal tree can be transformed by a body-fixed frame rotation, such that when it acts on one of the $X_{m}$-variables one of the following relations holds, depending on the position of the link,

$$
E_{l}^{a} X_{m}=-\frac{1}{2} \lambda^{a} X_{m_{3}}, \quad E_{l}^{a} X_{m}=\frac{1}{2} X_{m} \lambda^{a}, \quad E_{l}^{a} X_{m}=-\frac{1}{2} \lambda^{a} X_{m}+\frac{1}{2} X_{m} \lambda^{a} .
$$

In this last equation the first result holds if link $l$ is part of the path leading up to link $m$ from the origin, the second result holds if it is part of the path leading back back from link $m$ to the origin, and the third result holds if it is part of both paths. In this way the electric field operator generates long-range interactions between two link variables $X_{l}$ and $X_{l}$.

The links $W_{l}$ on the maximal tree change under local gauge transformations, and any function in the physical subspace must hence be a function only of the gauge-invariant $X_{l}$ variables. The gauge is effectively fixed by setting the $\operatorname{SU}(N)$ matrix operators $W_{l}$ to be the unit operator, so that we have $\left\{V_{l} ; W_{l}\right\} \rightarrow\left\{X_{l} ; 1\right\}$. It is now a matter of simple counting to show that the $X_{l}$ variables are precisely the (unconstrained) canonical variables of our Hamiltonian theory in the colourless sector, as summarised in Table 2 . On a finite lattice of length $n$ in $D$ dimensions there are $N_{s}=(n+1)^{D}$ lattice points, and $N_{l}=D n(n+1)^{D-1}$ links. The maximal tree contains $N_{t}=n+n(n+1)+n(n+1)^{2}+\cdots+n(n+1)^{D-1}=(n+1)^{D}-1=N_{s}-1$ 
Table 2. The number of degrees of freedom in the maximal tree approach on a hypercubic lattice of size $n \times n \times \cdots n$, for different number of space dimensions, D.

\begin{tabular}{l|ccc}
\hline Dimensionality, $D$ & 2 & 3 & $D \geq 2$ \\
\hline number of sites, $N_{s}$ & $(n+1)^{2}$ & $(n+1)^{3}$ & $(n+1)^{D}$ \\
number of links, $N_{l}$ & $2 n(n+1)$ & $3 n(n+1)^{2}$ & $D n(n+1)^{D-1}$ \\
number of links on tree, $N_{t}$ & $n+n(n+1)$ & $(n+1)^{3}-1$ & $(n+1)^{D}-1$ \\
number of independent $X_{l}$ & $n^{2}$ & $2 n^{3}+3 n^{2}$ & $D n(n+1)^{D-1}$ \\
variables, $N_{l}-N_{t}$ & & & $-(n+1)^{D}+1$ \\
\hline
\end{tabular}

links, as can easily be seen from (the $D$-dimensional generalisation of) our explicit choice of maximal tree. Therefore there are $N_{l}-N_{t}=D n(n+1)^{D-1}-(n+1)^{D}+1$ remaining links, and the same number of variables $X_{l}$. Each of these variables has $N^{2}-1$ degrees of freedom. Hence, the number of degrees of freedom of the $X_{l}$ variables is precisely equal to $N_{u}$, the number of unconstrained canonical degrees of freedom in the theory, as given by $\mathrm{Eq} .(20)$.

\subsection{The Hamiltonian in terms of the $X_{l}$ variables}

We now start with the Kogut-Susskind Hamiltonian of Eq. (19) and assume that it will act on a function of the variables $X_{l}$. For the electric piece of the Hamiltonian, $H_{E}$, we make use of the result in Eq. (21). In the magnetic part, $H_{M}$, we can set all of the links on the maximal tree to unity. After some algebra we arrive at a final expression for our Hamiltonian given entirely in terms of our complete set of (unconstrained) canonical variables, $X_{l}$. We do not quote the somewhat unedifying expression here. The interested reader is referred to Ref. [14] for further details and the full expression.

\section{Colour Neutrality and Further Constraints}

Although we have solved the major problem above of the constrained dynamics due to the gauge freedom, we still have to face a further problem of overcompleteness related to the number of degrees of freedom in the traces of $\mathrm{SU}(N)$ matrices. This leads to the existence of dependences among the traces of the Wilson loop variables, usually known as Mandelstam constraints. ${ }^{17}$

Our wave function in the pure gauge sector studied here should be a colour singlet, and the above complications arise when we impose colour neutrality on the wave function. Thus, the natural choice for us is now to work with traces of products of the variables $X_{l}$, as discussed above. A suitable approach would be to construct a basis of eigenstates of the electric part of the Hamiltonian, $H_{E}$, and calculate matrix elements of the magnetic energy between these states. Such an approach is a quite natural calculational scheme for the Hamiltonian approach. One can also use the method inherent in the Lagrangian calculations, which is based on invariant integration over the full group. ${ }^{18}$ However, for a proper Hamiltonian approach this 
discards many of the advantages of the method.

To find eigenstates of the electric operator, one can resort to three general approaches. Firstly, group theory gives us, in principle, a way to construct general eigenstates, the group characters. However, for a large basis, and $N>2$, this is extremely involved, ${ }^{19}$ unless it can be automated, and we see no easy way to do this.

A second approach is based on integrating configurations, and constructing orthogonal combinations from them. In this case one must start off with much larger overcomplete sets of configurations, and at increasing orders the integration, based on Creutz's integration method, ${ }^{20,21}$ tends to become more and more involved..$^{22}$

The third approach is based on the action of the electric operator itself, which leads to a block-diagonal matrix which has to be diagonalised to recover the eigenstates. In combination with a symbolic method explained more fully elsewhere, ${ }^{14}$ this seems to be the most powerful approach, which allows one to tackle any arbitrary $\mathrm{SU}(N)$ group. This is the method that we have developed and that we prefer to use with the maximal tree approach, although we note that it also has wider applicability. for further details the reader is referred to Ref. [14]

Our whole approach so far has been designed for applications in mind using a universal method of microscopic quantum many-body theory in the Hamiltonian formulation, such as the coupled cluster method $(\mathrm{CCM}) .{ }^{23}$ We do not have the scope in the present paper to discuss in any detail such many-body applications. Instead, we consider below the simpler limit where all plaquettes appear independently, and in so doing we introduce an extremely useful set of angular variables that considerably aid and simplify the analysis. We show that they serve to map an $\mathrm{SU}(N)$ one-loop problem onto an $N$-fermion problem on a torus.

\section{The One-Plaquette Problem}

If all of the loop variables are combined in such a way that they form plaquettes, ${ }^{21}$ we may consider our wave functional as depending only on the plaquette variables. Since the trace of a matrix is invariant under similarity transformations, we may write

$$
\operatorname{Tr}\left[U^{m}\right]=\operatorname{Tr}\left[\left(V U V^{-1}\right)^{m}\right]=\sum_{j=1}^{N} \mathrm{e}^{i m \phi_{j}} ; \quad m \in[1,2, \cdots, N],
$$

where $V$ diagonalises the $\mathrm{SU}(N)$ unitary matrix $U$ of the one-plaquette problem. Hence, out of the $N^{2}-1$ degrees of freedom for $\mathrm{SU}(N)$ the trace depends only on its $N$ eigenvalues $\exp \left(i \phi_{j}\right)$, all of which lie on the unit circle in the complex plane. The angular variables $\phi_{j}$ satisfy the constraint $\sum_{j=1}^{N} \phi_{j}=0$ coming from the $\operatorname{SU}(N)$ condition, $\operatorname{det} U=1$.

The wave functions of the one-plaquette problem can be expressed in a basis of group characters ${ }^{24}$ labelled by the standard $\operatorname{SU}(N)$ partitions $\lambda \equiv\left[\lambda_{1}, \lambda_{2}, \cdots, \lambda_{N}\right]$. These are simply proportional to the eigenstates of the electric operator (which 
is just the quadratic Casimir operator) for the single plaquette. We denote the group characters of $\mathrm{SU}(N)$ as $\chi_{\lambda}(\phi)$, where we use the shorthand notation $\phi \equiv$ $\left[\phi_{1}, \phi_{2}, \cdots, \phi_{N}\right]$. They may be represented as

$$
\chi_{\lambda}(\phi)=\frac{\epsilon\left(\lambda_{1}, \lambda_{2}, \cdots, \lambda_{N}\right)}{\epsilon((N-1),(N-2), \cdots, 2,1,0)} ; \quad \lambda_{1}>\lambda_{2}>\cdots>\lambda_{N}=0,
$$

where $\epsilon(\lambda)$ is the determinant of the $N \times N$ matrix $M$ with elements given by $M_{k l}=\exp \left(i \lambda_{k} \phi_{l}\right)$. The determinant in the denominator is a Vandermonde determinant of the eigenvalues of $U$, namely $\Delta \equiv \epsilon((N-1),(N-2), \cdots, 2,1,0)=$ $\prod_{k<l=1}^{N}\left[\exp \left(i \phi_{k}\right)-\exp \left(i \phi_{l}\right)\right]$. Its presence and its antisymmetry properties in the angular-variable eigenvalues allow us to map the original one-plaquette problem, which is symmetric under the interchange of the eigenvalues (i.e., an effective bosonic problem) but which has a complicated integration measure, into a much simpler fermionic problem by mapping $\Psi_{\lambda} \rightarrow \Phi_{\lambda} \equiv \Delta \Psi_{\lambda}$. In the electric part of the Hamiltonian we write correspondingly,

$$
\sum_{a=1}^{N^{2}-1} E^{a} E^{a} \rightarrow \Delta\left(\sum_{a=1}^{N^{2}-1} E^{a} E^{a}\right) \frac{1}{\Delta}=D_{N}-\frac{N\left(N^{2}-1\right)}{24},
$$

where the differential operator $D_{N}$, given by

$$
D_{N}=-\frac{1}{2} \sum_{i=1}^{N} \frac{\partial^{2}}{\partial \phi_{i}^{2}}+\frac{1}{2}\left(\frac{1}{\sqrt{N}} \sum_{i=1}^{N} \frac{\partial}{\partial \phi_{i}}\right)^{2}
$$

acts on the antisymmetric wave function $\Phi$.

When the the term $\operatorname{Tr}[U]+\operatorname{Tr}\left[U^{-1}\right]$ in the magnetic part, $H_{M}$, of the Hamiltonian in Eq. (19), acts on a wave function, it now reduces to a multiplication ${ }^{24}$ of group characters,

$$
\left[\chi_{1}(\phi)+\chi_{1}(-\phi)\right] \epsilon_{\lambda}=\sum_{\lambda^{\prime}=\lambda+1} \epsilon_{\lambda^{\prime}}+\sum_{\lambda^{\prime}=\lambda-1} \epsilon_{\lambda^{\prime}},
$$

where the symbols $\lambda \pm 1$ in the sums denote the inclusion of all possible partitions in which one and only one of the $\lambda_{i} \rightarrow \lambda_{i} \pm 1$ (with $1 \leq i \leq N$ ), while still fulfilling the requirements in Eq. (23). The operators $\operatorname{Tr}[U]$ and $\operatorname{Tr}\left[U^{-1}\right]$ thus act as raising and lowering operators, which act in a simple way on the Young tableaux corresponding to the group characters or wave functions with specific symmetry properties. ${ }^{14}$

We see from the above results that for a single $\mathrm{SU}(N)$ loop comprising $L$ links (where $L=4$ for a plaquette), the electric operator, $H_{E}$, in the Hamiltonian essentially yields $L$ times the difference between the total kinetic energy of the $N$ particles (now fermions) on a torus (represented by the angular variables $\phi_{i} ; i=1,2, \cdots, N$ ) and their centre-of-mass energy. It is very naturally expressed in terms of the momentum operators $p_{j} \equiv-i \frac{\partial}{\partial \phi_{j}}$. The magnetic term, $H_{M}$, for the single plaquette is also easily seen to be given as $2 \lambda \sum_{j=1}^{N}\left(1-\cos \phi_{j}\right)$. The constraint $\operatorname{det} U=1$ now reduces to a constraint on the centre-of-mass motion, $\Phi \equiv N^{-1} \sum_{j=1}^{N} \phi_{j}=0$. We need 


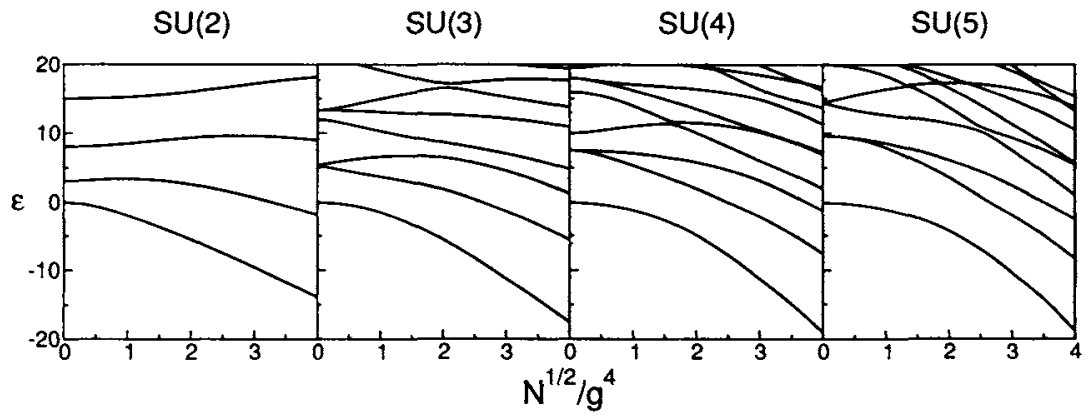

Fig. 2. The lowest levels of the one-plaquette energy spectra for SU(2) to SU(5).

also to impose the constraint on the centre-of-mass momentum, $P \equiv \sum_{j=1}^{N} p_{j}=0$, since $\Phi$ is an unphysical variable for the $\mathrm{SU}(N)$ problem. A straightforward application of the Dirac quantisation procedure to handle the constraints then easily leads to the following realisation of the one-plaquette Hamiltonian,

$$
H=\frac{g^{2}}{2 a}\left(2 \sum_{j=1}^{N}\left[p_{j}-P / N\right]^{2}+2 \lambda \sum_{j=1}^{N}\left[1-\cos \left(\phi_{j}-\Phi\right)\right]\right),
$$

which we have written for the physical case $D=3$ for which $\lambda \equiv g^{-4}$. In this form the Hamiltonian is now manifestly translationally invariant. In the weak-coupling limit $(g \rightarrow 0)$ (or, equivalently, $\lambda \rightarrow \infty$ ) we may readily use the harmonic approximation for the magnetic ("potential energy") term of the $N$-fermion problem. In this limit the entire centre-of-mass energy factorises. This great simplification allows for the easy implementation of the constraint $\operatorname{det} U=1$, and the degeneracies $\nu_{n}$ of the $n$th level of the equidistant spectrum are now given by $\nu_{n}=P_{N}(n)-P_{N}(n-1)$, where $P_{m}(n)$ is the number of different ways the positive integer $n$ can be partitioned as the sum of $m$ positive integers.

\subsection{Numerical results}

We now investigate the one-plaquette spectra of a few of the relevant $\mathrm{SU}(N)$ gauge theories, namely for $2 \leq N \leq 5$. The method we use to solve the problem is first to work in a basis of eigenstates of the electric Hamiltonian, and then to evaluate the action of $\operatorname{Tr}[U]$ and $\operatorname{Tr}\left[U^{-1}\right]$ on these states. For the low-lying spectra that we calculate we solve the linear eigenvalue equations numerically. From the form of the Hamiltonian in Eq. (19) or Eq. (27), we see that it is convenient to define a scaled energy $\varepsilon$ as follows,

$$
E=\frac{g^{2}}{2 a} \varepsilon+\frac{N}{g^{2} a},
$$

which we use for representing the numerical results. 
For SU(2), the one-plaquette Schrödinger equation reduces to the Mathieu equation and the spectrum is given by its odd characteristic values, as shown in the left panel of Fig. 2. No such closed-form analytical solutions seem to exist for $\mathrm{SU}(N)$ with $N>2$, and we resort to the numerical procedure described above to obtain the remaining results shown in Fig. 2. We note that the spectra for $N>2$ are much richer than for $N=2$. They include not only many avoided crossings but also, very interestingly, what appear to be several real crossings in the cases $N=4$ and $N=5$. We have verified that the distances between the respective two levels in these latter cases are equal to zero within our numerical accuracy. Such real crossings, of course, are a reflection of some (otherwise hidden) symmetry in these theories. They clearly deserve further investigation.

Finally, we note that the region of the coupling constant shown in Fig. 2 is insufficient to observe the asymptotic convergence to the harmonic approximation discussed above. However, we have checked numerically, by going to values $g^{-4} \geq$ 50 , that our stated results for the degeneracy factors for the equidistant harmonic oscillator spacings in this limit are correct.

\subsection{Independent-plaquette wave functionals}

The results for the one-plaquette problem have more consequences for more general (e.g., variational or CCM) wave functionals than one might at first suspect. Thus, if the trace variables, $\xi_{m} \equiv m^{-1} \operatorname{Tr}\left(U^{m}\right)$, of the one-plaquette matrix are used, the wave functional is a function of the group characters only. The specific wave functional that is the sum of one-plaquette functions,

$$
\left\langle\left\{\phi_{j}^{\alpha}\right\} \mid \Sigma\right\rangle=\sum_{\text {plaquettes } \alpha} F\left(\left\{\phi_{j}^{\alpha}\right\}_{j=1}^{N}\right),
$$

naturally leads to the sum of one-plaquette problems, leading to total energies which are the sum of one-plaquette energies. However, the corresponding product wave functional

$$
\left\langle\left\{\phi_{j}^{\alpha}\right\} \mid \Pi\right\rangle=\prod_{\text {plaquettes } \alpha} F\left(\left\{\phi_{j}^{\alpha}\right\}_{j=1}^{N}\right)
$$

also leads to the same result as we now show. This absence of correlations between nearest-neighbour plaquettes, follows in our case from the symmetry of (the original bosonic) wave functional, $\Psi_{\lambda}$, in the angular variables,

$$
\Psi_{\lambda}\left(\cdots \phi_{i} \cdots \phi_{j} \cdots\right)=\Psi_{\lambda}\left(\cdots \phi_{j} \cdots \phi_{i} \cdots\right)
$$

where we decompose

$$
F\left(\phi_{1} \cdots \phi_{N}\right)=\sum_{\lambda} c_{\lambda} \Psi_{\lambda}\left(\phi_{1} \cdots \phi_{N}\right)
$$

Therefore the cross-product term from the electric operator vanishes,

$$
\begin{aligned}
& D_{N} \Psi_{\lambda}^{\prime}\left(\phi_{1}^{\alpha} \cdots \phi_{N}^{\alpha}\right) \Psi_{\lambda^{\prime}}^{\prime}\left(\phi_{1}^{\beta} \cdots \phi_{N}^{\beta}\right) \\
& =\Psi_{\lambda^{\prime}}^{\prime}\left(\phi_{1}^{\beta} \cdots \phi_{N}^{\beta}\right) D_{N} \Psi_{\lambda}^{\prime}\left(\phi_{1}^{\alpha} \cdots \phi_{N}^{\alpha}\right)+\Psi_{\lambda}^{\prime}\left(\phi_{1}^{\alpha} \cdots \phi_{N}^{\alpha}\right) D_{N} \Psi_{\lambda^{\prime}}^{\prime}\left(\phi_{1}^{\beta} \cdots \phi_{N}^{\beta}\right),
\end{aligned}
$$


where $\alpha$ and $\beta$ are plaquettes containing the link $l$, and $\Psi_{\lambda}^{\prime}=\operatorname{det}(J) \Psi_{\lambda}$, where $\operatorname{det}(J)=|\Delta|^{2}$ is the Jacobian of the transformation from trace variables to angular variables, $J_{m j} \equiv \partial \xi_{m} / \partial \phi_{j}=i \exp \left(i m \phi_{j}\right)$. The differential operator $D_{N}$ contains both sets of angular operators, $\partial_{\phi_{i}}=\partial_{\phi_{i}^{\alpha}}+\partial_{\phi_{i}^{\beta}}$. Therefore the Hamiltonian, when acting on the product wave functional $|\Pi\rangle$, also reduces to the sum of one-plaquette Hamiltonians.

\section{Summary and Whither Next?}

In summary, we have shown how to fix the gauge by using the maximal-tree gauge, which is specific to the lattice versions of gauge field theories. It does not suffer from the typical problems of gauge fixing in the continuum. We then introduced a very natural set of angular variables, simply related to the trace (or Wilson loop) variables for a fundamental plaquette, in terms of which the $\mathrm{SU}(N)$ one-loop problem can be mapped onto an $\mathrm{N}$-fermion problem on a torus. Exact solutions were obtained for a single plaquette in both the weak-coupling $(g \rightarrow 0)$ and strongcoupling $(g \rightarrow \infty)$ limits. The eigenstates in the strong-coupling limit are the group characters of the corresponding group, and in the weak-coupling limit the harmonic approximation to the fermion problem leads to explicit formulas for the degeneracies of the equidistant energy levels. In terms of the group characters (i.e., the eigenstates of the electric piece of the Hamiltonian), the ground and low-lying energy states of the single plaquette can be determined numerically for arbitrary values of the coupling constant, since the Hamiltonian reduces to a simple linear equation in terms of these group characters. The terms in the magnetic piece of the Hamiltonian are simple raising and lowering operators in this basis, and hence the energy spectra are found by a simple configuration-interaction method technique of diagonalising the resultant block-diagonal Hamiltonian matrix in a restricted basis. The procedure can be automated using the theory of multiplication of group characters, in terms of the Young tableaux of the corresponding group.

Another, somewhat unexpected, advantage of our approach is the fact that correlations between spatially distinct trace variables cancel. This leads to the intriguing possibility that spatial correlations in the full lattice problem might be weak in our approach, which would in turn tend to suggest that successive approximations in a fully consistent microscopic many-body approach based on our approach might be expected to converge rapidly. Clearly, the simplicity of the angular variables formulation is promising for more elaborate wave functionals. An obvious extension to include correlations is to employ the coupled cluster method (CCM), ${ }^{23}$ which has been extensively applied with great success to a wide variety of quantum many-body systems and quantum field theories. Typical applications of the CCM to strongly interacting continuum quantum field theories include $\phi^{4}$ field theory ${ }^{25,26}$ and a model field theory of pions and nucleons. ${ }^{27}$ There have been many applications of the CCM to various spin-lattice models in quantum magnetism. ${ }^{28}$ Other applications to lattice field theories include the $O(4)$ nonlinear sigma model as a model of meson field 
theory with a phase transition due to chiral symmetry breaking. ${ }^{29}$

Previous attempts to use the CCM in lattice gauge theory have largely been confined to the ground and low-lying excited states in the pure gauge (gluon) sectors of the $\mathrm{U}(1)$ and $\mathrm{SU}(2)$ cases in one and two spatial dimensions. ${ }^{1,2}$ As we have seen, for $D<3$ the plaquette variables $U_{p}$ form a natural complete set. However, they are overcomplete for $D \geq 3$. One of our key aims here has been to find a natural complete set of variables for this case. We have shown that the variables $X_{l}$ fulfil this role.

At the heart of the CCM is the parametrisation of the ket-state many-body wave function as an exponential of a correlation operator formed from a linear combination of mutually commuting creation (or excitation) operators with respect to a model or reference state $|\Phi\rangle$,

$$
|\Psi\rangle=\mathrm{e}^{S}|\Phi\rangle \quad ; \quad S=\sum_{I} c_{I}|I\rangle\langle\Phi|
$$

The creation operators here excite from the model state to an arbitrary excited state $|I\rangle$, and do not act between different excited states on overlapping lattice-site configurations.

From this vantage point the key question is then the choice of the form of the model state $|\Phi\rangle$ and of the states in which to expand the many-body Hilbert space. Clearly, the simplest choice for the model state is just the chromo-electric vacuum. As for the remainder, this is precisely the question that we have addressed here, where we have established the viability for such purposes of the set of independent maximal-tree variables, $X_{l}$, and their conjugate variables, $E_{l}^{a}$. Thus, for the colourless (pure gauge) sector we have to use closed contours, which are traces over products of $X_{l}$ variables, since only these variables are invariant under gauge transformations generated by Gauss' law.

We conclude with some final comments. Firstly, we note that the form of our $\mathrm{SU}(N)$ Hamiltonian in the $X_{l}$ variables is very different from the naive KogutSusskind form of Eq. (19). This is likely to have an important effect on the role of correlations in the ensuing analysis. Secondly, a price that we pay for using the maximal-tree gauge is that the tree, and hence our Hamiltonian, has a preferred direction and we thereby lose explicit translational invariance. Although this is presumably restored in a full CCM calculation carried out to all orders, in practice we need to make approximations via a systematic hierarchy of choices for which excited states $|I\rangle$ to include in the CCM ket-state correlation operator $S$ in Eq. (34), and it is difficult to know in advance how serious the loss of translational invariance might be in practice at attainable levels of implementation. An obvious next step in this regard would be to investigate the possibility of designing different, more symmetric, choices for the maximal tree in order to circumvent this problem. On the other hand, although the current choice might turn out to be problematic for a study of the vacuum sector, it would seem to be a natural choice for the study of the interaction between fixed sources on the lattice, which explicitly breaks translational 
invariance. Such studies are themselves an obvious next step in the extension of the current approach away from the pure gauge sector to include fermions. We note that some very preliminary steps in this direction have already been taken in the much simpler case of the Schwinger model of quantum electrodynamics in one spatial dimension, whose Hamiltonian form on the lattice has been investigated, using CCM techniques, with the inclusion of massive staggered fermions. ${ }^{30}$

\section{References}

1. R. F. Bishop and Y. Xian, Acta Phys. Pol. B24, 541 (1993); R. F. Bishop, A. S. Kendall, L. Y. Wong, and Y. Xian, Phys. Rev. D 48, 887 (1993); R. F. Bishop and Y. Xian, in Condensed Matter Theories, Vol. 9, eds. J. W. Clark, K. A. Shoaib, and A. Sadiq, (Nova Science Publ., Commack, N.Y., 1994), p. 433; S. J. Baker, R. F. Bishop, and N. J. Davidson, Phys. Rev. D 53, 2610 (1996).

2. C. H. Llewellyn Smith and N. J. Watson, Phys. Lett. B302, 463 (1993).

3. C. N. Yang and R. L. Mills, Phys. Rev. 96, 191 (1954).

4. L. D. Faddeev and A. A. Slavnov, Gauge Fields, Introduction to Quantum Theory (Benjamin Cummings, Reading, Mass., 1980).

5. P. A. M. Dirac, Principles of Quantum Mechanics, 4th ed., (Oxford University Press, Oxford, 1967).

6. P. A. M. Dirac, Lectures on Quantum Mechanics, (Belfer Graduate School of Science, Yeshiva University, New York, 1964); Lectures on Quantum Field Theory, (Belfer Graduate School of Science, Yeshiva University, New York, 1966).

7. T. Muta, Foundations of Quantum Chromodynamics, (World Scientific, Singapore, 1987).

8. M. Henneaux and C. Teitelboim, Quantization of Gauge Systems, (Princeton University Press, Princeton, 1992).

9. J. Kogut and L. Susskind, Phys. Rev. D 11, 395 (1975).

10. J. B. Kogut, Rev. Mod. Phys. 51, 659 (1979).

11. F. A. Berezin, The Method of Second Quantization (Academic Press, New York, 1966).

12. B. Felsager, Geometry, Particles and Fields (Odense University Press, Odense, 1981).

13. G. 't Hooft and M. Veltman, Nucl. Phys. B44, 189 (1972).

14. N. E. Ligterink, N. R. Walet, and R. F. Bishop, Ann. Phys. (N.Y.) 284, 215 (2000).

15. V. F. Müller and W. Rühl, Nucl. Phys. B230, 49 (1984).

16. J. B. Bronzan, Phys. Rev. D 31, 2020 (1985); ibid. 37, 1621 (1988); ibid. 38, 1994 (1988); J. B. Bronzan and T. E. Vaughan, ibid. 43, 3499 (1991); ibid. 47, 3543 (1993).

17. S. Mandelstam, Phys. Rev. D 19, 2391 (1979).

18. D. Horn and M. Karliner, Nucl. Phys. B235,135 (1984).

19. M. A. B. Bég and H. Ruegg, J. Math. Phys. 6, 677 (1965).

20. M. Creutz, J. Math. Phys. 19, 2043 (1974).

21. M. Creutz, Quarks, Gluons, and Lattices, (Cambridge Univ. Press, Cambridge, 1983).

22. C. R. Leonard, Ph.D. thesis, Melbourne University, 1998.

23. R. F. Bishop, Theor. Chim. Acta 80, 95, (1991); in Microscopic Quantum ManyBody Theories and Their Applications, eds. J. Navarro and A. Polls, Lecture Notes in Physics, Vol. 510, (Springer-Verlag, Berlin, 1998), p. 1.

24. H. Weyl, The Classical Groups, 2nd ed., (Princeton Univ. Press, Princeton, 1946); J. E. Hetrick, Int. J. Mod. Phys. A 9, 3153 (1994); J. Hallin, Class. Quant. Grav. 11, 1615 (1994).

25. U. Kaulfuss, Phys. Rev. D 32, 1421 (1985); C. S. Hsue, H. Kümmel, and P. Ueber- 
holz, ibid. 32, 1435 (1985); M. Altenbokum and H. Kümmel, ibid. 32, 2014 (1985); M. Funke, U. Kaulfuss, and H. Kümmel, ibid. 35, 621 (1987).

26. A. H. Rezaeian and N. R. Walet, J. High Energy Phys. 12, 040 (2003); Phys. Lett. B570, 129 (2003).

27. H. Kümmel, Phys. Rev. C 27, 765 (1983); G. Hasberg and H. Kümmel, ibid. 33, 1367 (1986).

28. R. F. Bishop, J. B. Parkinson, and Y. Xian, Phys. Rev. B 44, 9425 (1991); C. Zheng, D. J. J. Farnell, and R. F. Bishop, J. Stat. Phys. 60, 327 (1998); J. Rosenfeld, N. E. Ligterink, and R. F. Bishop, Phys. Rev. B 60, 4030 (1999); S. E. Krüger, J. Richter, J. Schulenburg, D. J. J. Farnell, and R. F. Bishop, ibid. 61, 14607 (2000); D. J. J. Farnell, R. F. Bishop, and K. A. Gernoth, J. Stat. Phys. 108, 401 (2002); D. J. J. Farnell and R. F. Bishop, in Quantum Magnetism, eds. U. Schollwöck, J. Richter, D. J. J. Farnell and R. F. Bishop, Lecture Notes in Physics, Vol. 645, (Springer-Verlag, Berlin, 2004), p. 307.

29. N. E. Ligterink, N. R. Walet, and R. F. Bishop, Ann. Phys. (N.Y.) 267, 97 (1998); Phys. Rev. E 63, 037103 (2001).

30. R. McDonald and N. R. Walet, Int. J. Mod. Phys. B 17, 5393 (2003). 